\title{
Growth and Characterization of Pure and L-lysine Doped Zinc (TRIS) Thiourea Sulphate Crystals
}

\author{
Dr. J. Thomas Joseph Prakash \\ Assistant Professor, Department of Physics, \\ H.H. The Rajah's Government Arts College, \\ Pudukkottai, Tamil Nadu, India. \\ E-mail: armyjpr1@yahoo.co.in
}

\author{
M. Lawrence \\ Assistant Professor, \\ Department of Physics, \\ Kurinji College of Arts and Science, \\ Tiruchirappalli, Tamil Nadu, India
}

\begin{abstract}
Single crystals of Zinc (tris) thiourea sulphate (ZTS) doped with basic amino acid l-lysine were grown successfully by slow evaporation method at ambient temperature. The concentration of dopants in the mother solution was varied from $0.1 \mathrm{~mol} \%$ to $0.5 \mathrm{~mol} \%$. The solubility data for all dopants concentration were determined. There is a drastic change in morphology due to doping which is also reflected in the Xray diffraction pattern. The Fourier transform infrared spectroscopy study confirms the incorporation of 1-lysine into ZTS crystal. The doped crystals are optically better and more transparent than the pure ones having wide transmission spectra lying between 240 and $1200 \mathrm{~nm}$. The nonlinear optical (NLO) property of the grown crystal has been confirmed by Kurtz-power second harmonic generation (SHG) test. The dopants increase the hardness value of the material and it also depends on the concentration of the dopants. Results are discussed.
\end{abstract}

Keywords: Crystal morphology; X-ray diffraction; Growth from solutions; Zinc compounds; Nonlinear optic materials

\section{INTRODUCTION}

In recent years several studies dealing with organic, inorganic and semiorganic molecules and materials called nonlinear optics (NLO) are being reported, due to the increasing need for cheap and easily processable materials for photonics applications. The nonlinear (NLO) responses induced in various molecules in solution and solids are of great interest in many fields of research $[1,6]$. Thiourea molecules are an interesting inorganic matrix modifier due to its large dipole moment and its ability to form and extensive network of hydrogen bonds [8]. The nonlinear optical properties of some of the complexes of thiourea, such as bis (thiourea) cadmium chloride (BTCC), bis (thiourea) zinc chloride (BTZC), tris (thiourea) zinc sulphate (ZTS), tris (thiourea) cadmium sulphate (CTS), potassium thiourea bromide (PTB) have gained significant attention in the last few years [13, $16 \& 25]$, because both organic and inorganic components in it contribute specifically to the process of second harmonic generation. The centrosymmetric thiourea molecule, when combine with inorganic salt yield noncentrosymmetric complexes, which has the nonlinear optical properties [3]. Hence, in several years, search is focused on new types NLO materials which combined the advantages of organic and inorganic material called semiorganic materials. Two types of semiorganic material include organic and inorganic salts and metal organic coordination complexes [14, 17, 18, 19, 21 \& 22]. Zinc (tris) thiourea sulphate (ZTS) is a good nonlinear optical semiorganic material for second harmonic generation. ZTS has high laser damage threshold, low angular sensitivity, wide rang of transparency and low dielectric constant at high frequencies [5]. ZTS is 1.2 times more nonlinear than KDP [23]. ZTS possesses orthorhombic structure with Pca2 $2_{1}$ space group [15]. The growth and various studies of doped and undoped ZTS crystals have been reported in a number of publications $[4,7,9,10,12,20, \& 24]$. In this paper we report the results of our work on the growth of pure and amino acid (L-lysine) doped ZTS crystal along with the characterization by X-ray diffraction (XRD), Fourier Transform Infrared (FTIR), UV-Visible study, and Microhardness techniques.

\section{EXPERIMENTAL}

2.1. Synthesis and crystal growth

The ZTS salt was synthesized by dissolving high purity AR grade Zinc sulphate and thiourea in molar ratio 1:3 in deionized water. The solution was stirred by magnetic stirrer. White crystalline ZTS salt was obtained immediately. ZTS salt was synthesized according to the reaction.

$$
\left.\mathrm{ZnSo}_{4}+3 \mathrm{CS}\left(\mathrm{NH}_{2}\right)_{2} \rightarrow \mathrm{Zn}\left[\mathrm{CS}\left(\mathrm{NH}_{2}\right)_{2}\right) 3\right] \mathrm{SO}_{4}
$$

Temperature was maintained at $50{ }^{\circ} \mathrm{C}$ to avoid decomposition. Single crystals of ZTS and L-lysine doped ZTS were grown slow evaporation techniques at room temperature $\left(32^{\circ} \mathrm{C}\right)$. Transparent colorless ZTS crystals of size $15 \times 12 \times 2 \mathrm{~mm}^{3}$ were harvested in 18 days. For the growth of L-lysine doped ZTS crystals, $1 \mathrm{~mol}$ percent of L-Lysine was added to the solution of ZTS. Single crystals of size $10 \times 9 \times 2 \mathrm{~mm}^{3}$ with good transparency were harvested in 30 days as shown in Figure 1.

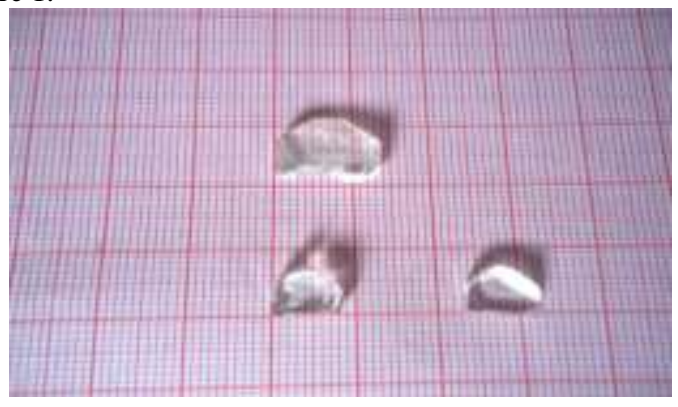

Fig. 1. The photograph of grown pure ZTS and L-Lysine doped ZTS crystals 


\section{CHARACTERIZATION}

The grown crystals have been analyzed by different characterization techniques. The Grown single crystal of pure and amino acid doped ZTS was confirmed by single crystal X-ray diffraction analysis using ENRAF NONIUS CAD4 diffractometer. The functional groups were identified by using PERKIN ELMER RX1 Fourier Transform Infrared spectrophotometer in the range of $400-4000 \mathrm{~cm}-1$. The optical properties of the crystals were examined between 200 and $1200 \mathrm{~nm}$ using LAMBDA-35 UV-Vis spectrometer. The NLO efficiency of the grown sample confirmed Nd: YAG laser as the source. The mechanical property of the grown crystals has been studied using a Leitz Weitzler hardness tester fitted with a diamond pyramidal indenter.

\subsection{Single crystal $X$-ray diffraction analysis}

The single X-ray diffraction studies have been carried out to confirm the crystallinity and to determine the lattice parameters of the grown sample. The single crystal X-ray diffraction has been carried out using ENRAF NONIUS CAD4 diffractrometer. The structure was solved by the direct method using SHELXL program. From the XRD data it is observed that both pure and doped L-lysine crystals are orthorhombic. The calculated lattice parameter values of pure and L-lysine doped ZTS are presented in table 1 . The results of the present work are in good agreement with the reported values [2]. In the case of doped sample, a slight variation in the cell volume is observed.

Table 1 Single-crystal XRD data pure and L-lysine doped ZTS crystals

\begin{tabular}{lcc}
\hline & Pure ZTS & L-lysine doped ZTS \\
Cell parameters & $\mathrm{a}=7.797 \AA$ & $\mathrm{a}=7.789 \AA$ \\
& $\mathrm{b}=11.144 \AA$ & $\mathrm{b}=11.150 \AA$ \\
& $\mathrm{c}=15.512 \AA$ & $\mathrm{c}=15.517 \AA$ \\
& \multicolumn{2}{c}{$\alpha=\beta=\gamma=90^{\circ}$} \\
Volume & $\mathrm{V}=1348 \AA^{3}$ & $\mathrm{~V}=1347.5 \AA^{3}$ \\
System & & \multicolumn{2}{c}{ Orthorhombic } \\
Space group & $\mathrm{Pca}_{1}$ &
\end{tabular}

\subsection{FTIR studies}

FTIR spectrum of pure and L-lysine doped ZTS crystal was in the $\mathrm{KBr}$ pellet technique in the frequency region $400-4000 \mathrm{~cm}-1$ using PERKIN ELMER RX1 Fourier Transform Infrared spectrometer. The recorded FTIR spectrum of pure and L-lysine doped ZTS crystal is shown in Figure 2. The broad band lying in the range $2710-3377 \mathrm{~cm}-1$ corresponds to symmetric and asymmetric vibrations of $\mathrm{NH}_{2}$ group. Very strong absorption at $946 \mathrm{~cm}-1$ is due to sulphate ion. The FTIR spectra of doped crystals show a strong $\mathrm{NH}$ absorption peak at about $3200 \mathrm{~cm}-1$. When ZTS is doped with L-lysine more NH stretch vibrations are introduced due to doping and as a result the $\mathrm{NH}$ absorption peak becomes stronger. So FTIR spectra indirectly establish the presence of amino acid, L-lysine in the lattice of ZTS crystal.

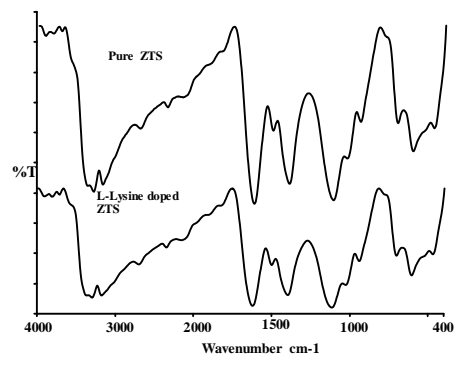

Fig. 3. FTIR spectra of pure and L-lysine doped ZTS

\subsection{Optical transmission studies}

The UV-VIS spectrum analysis has been measured using LAMBDA-35 UV-Vis spectrophotometer in the wavelength range of $200-1200 \mathrm{~nm}$. The UV-VIS spectrum gives limited information about the structure of the molecule because the absorption of UV and visible light involves promotion of the electron in $\sigma$ and $\pi$ orbital from the ground state to higher energy states. To find the transmission range of ZTS, the optical transmission spectrum of the ZTS for the wavelengths between 200 and $1200 \mathrm{~nm}$ was recorded. The recorded optical transmission spectrum was shown in Figure 4. The transmittance is found to be maximum in the entire visible and infrared regions. When we consider the percentage of transmission we observe that for all L-lysine doped ZTS crystals the transmission has been increased $75 \%$ in the visible region. From the spectrum it is observed that the transmittance percentage of doped ZTS is $15 \%$ higher than that of the pure grown crystal. The crystal shows a good transmittance in the entire visible region. The lower cutoff at $280 \mathrm{~nm}$ combined with the above, attest the usefulness of this material for optoelectronics applications and the second harmonic generation of the Nd:YAG laser and for the generation of the higher harmonics of the laser diodes. A good optical transmittance is very desirable in a nonlinear optical (NLO) crystal since the absorptions, if any, in an NLO material near the fundamental or the second harmonic of an Nd: YAG laser, $1064 \mathrm{~nm}$ and $532 \mathrm{~nm}$ respectively, will lead to loss of conversion efficiency of second harmonic generation (SHG).

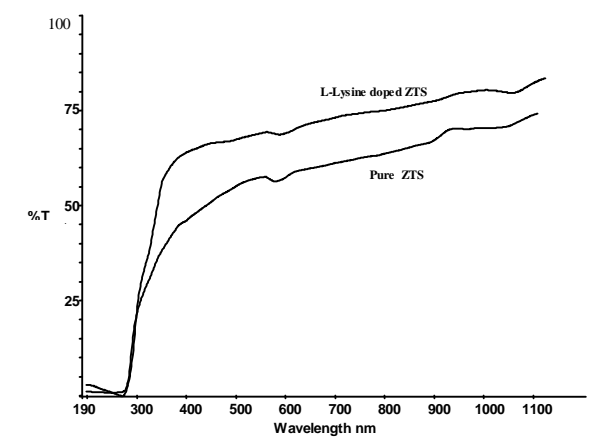

Fig. 4. UV Vis spectra for the pure and L-lysine doped ZTS

\subsection{Second harmonic generation (SHG) efficiency}

The second harmonic generation (SHG) conversion efficiency of ZTS was measured by powder Kurtz and Perry powder technique [11]. The crystal was grounded into a fine powder and densely packed between two transparent glass slides. A Q switched Nd: YAG laser emitting a fundamental wavelength of $1064 \mathrm{~nm}$ (pulse width $8 \mathrm{~ns}$ ) was allowed to strike the sample cell. The SHG output $532 \mathrm{~nm}$ (green light) was finally detected by the photomultiplier tube. The powdered material of potassium dihydrogen phosphate (KDP) was used in the same experiment as a reference material. Second harmonic generation efficiency of the doped L-lysine crystal almost same as the pure ZTS

\subsection{Microhardness measurement}

The definition of hardness depends entirely on the method of measurement which will determine the scale of hardness obtained. The best general definition that can be given is that hardness is a measure of the resistance deformation. An 
important use of microhardness study is the possibility of making an indirect estimate of other mechanical characteristics of materials having a specific correction with their hardness. It plays a key role in device fabrication. Transparent crystals free from cracks were selected for microhardness measurements. There are different types of hardness tests available viz., static indentation test, dynamic indentation test scratch test, rebound test, pendulum recoil test, in which Vicker's Microhardness studies has been used for the present study The mechanical property of the grown crystals has been studied using a LEITZ microhardness tester fitted with a Vickers diamond pyramidal indenter. A well polished ZTS crystal was placed on the platform of Vickers microhardness tester and the loads of different magnitudes were applied over a fixed interval of time. The indentation time was kept $(8 \mathrm{~s})$ for all the loads. The hardness number was calculated using the relation

$$
\mathrm{H}_{\mathrm{v}}=\frac{(1.8544 * \mathrm{P})}{\mathrm{d}^{2}} \mathrm{~kg} / \mathrm{mm}^{2}
$$

Where Hv is the Vickers microhardness number, $\mathrm{P}$ is the applied load in $\mathrm{kg}$ and $\mathrm{d}$ is the diagonal length of the indentation impression in the micrometer. A graph has been plotted between hardness number $(\mathrm{Hv})$ and applied load (P) as shown in Figure 5. The hardness increased gradually with the increase of load. L-lysine, the increase in hardness is large and at higher concentration of impurity the hardness tends to saturate. The possible explanation for this behavior is as follows. In the solid state L-lysine exists as zwitterions. So introduction of L-lysine in the crystal lattice of ZTS creates ionic vacancy. All these defects, act as obstacles to dislocation motion, thus increasing the hardness of the crystals. At higher concentration of impurity, the impurity-vacancy associates into larger aggregate. So, hardness saturates at higher concentration of impurity.

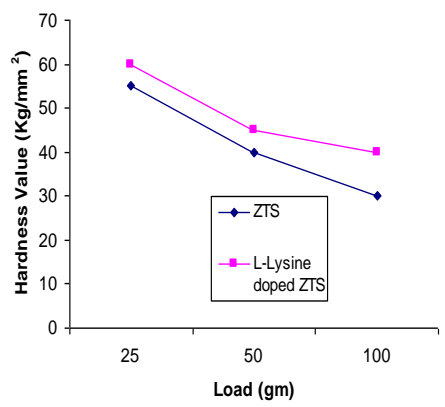

Figure 5.Variation of Vicker's microhardness number with applied load

\subsection{Etching studies}

To obtain the structural perfection and growth features of a grown crystal, etching studies can be used. The (100) plane of the pure and doped ZTS crystal has been completely immersed for $10 \mathrm{~s}$ in the water etchant and the sample was wiped out with dry filter paper. Using a Magnus MLX Microscope the features of the crystal has been analyzed which is shown in the Figure 6(a), 6(b).From the figures, number of etch pits have been identified with identical shape. By increasing the etching time, the pattern remains the same but the size of the etch pits have been increases. The etch pit can be attributed to the initial dislocations formed at low angle boundaries or segregated impurities.

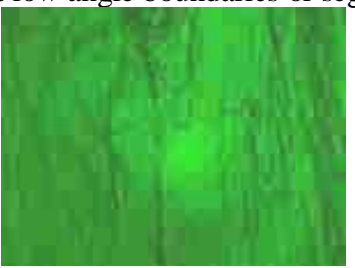

Etch patterns on Fig. 6 (a) pure ZTS - $10 \mathrm{~s}$

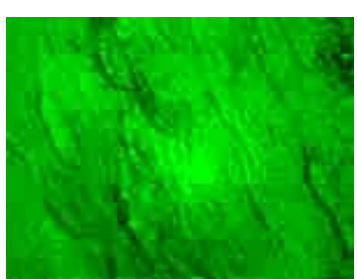

Etch patterns on Fig 6 (b) L-lysine doped ZTS - $10 \mathrm{~s}$

\section{CONCLUSION}

Good optical quality pure ZTS and L-lysine doped ZTS single crystals have been grown by solution growth method at room temperature. The lattice parameters have been found by single crystal X-ray diffraction technique. The FT-IR spectrum reveals that the various functional groups present in the grown crystal. The optical absorption spectrum reveals that the absorbance is less than one unit between 300 and $1200 \mathrm{~nm}$. This illustrates the absence of any overtones or combination modes above $250 \mathrm{~nm}$ and absorbance due to electronic transition between 300 and $1100 \mathrm{~nm}$. The Vicker's microhardness was calculated in order to understand the mechanical stability of the grown crystals. Hardness measurement also shows that L-lysine doped crystals are much harder than pure ZTS crystals. The studies on the NLO property confirmed the second harmonic conversion efficiency of the crystal to be better than KDP. The crystal perfections and quality have been identified by using the etching studies.

\section{REFERENCES}

[1] Agggarwal, M.D., Choi, J., Wang, W.S., Bhat, K., Lal, R.B., Shield, A.D., Penn, B.G., Frazier, D.O. J.Crystal Growth 204 (1999)179.

[2] Andreeti, G.D., Cavalca, L., Musatti, A. Acta Crystallogr, sect. B 24 (1968) 683.

[3] Anie Roshan, S., Joseph, C., Ittachen, M.A. Matter.Lett.49 (2001)299.

[4] Dhumane, N.R., Hussaini, S.S., Dongre, V.G., Mahendra, Shirsat, D. Optical Materials, Volume 31, Issue 2, October-December 2008, Pages 328-332

[5] Dhumane, N.R., Hussaini, S.S., Nawakhele, V.V., Shirsat, M.D. Cryst. Res. Technol. 41 (2006)897.

[6] Elmert, D., Velsko, S., Davis, L., Wang, F., Loiaceono, G., Kennedy, G. IEEE. J. Quantum Electron. 25 (1989) 179.

[7] Gupte Sonal S. Pradhan Ranjit D. J Appl Phys 2002; 91: $3125-8$

[8] Hellwege, K.H., Hellwage, A.M. Landolt-Bornstein Group I I 14 (1982) 584.

[9] Krishnan, C., Selvarajan, P., Freeda, T.H. Journal of Crystal Growth, Volume 311, Issue 1, 15 December 2008, Pages 141-146

[10] Krishnan, C., Selvarajan, P., Freeda, T.H., Mahadevan, C.K. Physica B: Condensed Matter, Volume 404, Issue 2, 28 February 2009, and Pages289-294

[11] Kurtz, S.K., Perry, T.T., J. Appl. Phys., 39 (1968) 3798. 
[12] Marcy Ho, Warren LF, Webb MS, Ebbers CA, Velsko SP, Kenndy GC, et al. J Appl Opts 1992; 31: 5051-60

[13] Marcy, H.O., Warren, L.F., Hebb, M.S., Ebbers, C.A., Visko, S.P., Kennedy, C.G. J. Appl.Opt. 31 (1992)5051.

[14] Meera, K., Muralidharan, R., Dhanasekaran, R., Prapun Manyum, Ramasamy, P. J.cryst. Growth 263 (2004)510.

[15] Oussaid, M., Becker, P., Carabatos Nedelac, C. Phys. Status Solidi B 207 (1998) 499.

[16] Oussaid, M., Becker, P., Kemiche, M. C. Carabatos-Nedlec, Phs. Stat. Sol.B 207 (1998) 103.

[17] PricillaJeyakumari, A., Ramajothi, J., Dhanuskodi, S., J.Cryst.Growth 269 (2004)558.

[18] Rajasekaran, R., Ushasree, P.M., Jayavel, R., Ramasamy, P. J.cryst. Growth 229 (2001)563.

[19] Ramajothi, J., Dhanuskodi, S., Nagarajan, K. Cryst. Res. Technol. 39 (2004) 414.
Sumil Verma, Singh Wadhawan, MK. Suresh, VK., Pramana, CH. J Phys 2000; 54:879-88.

[21] Sun, H.Q., Yuan, D.R., Wang, X.Q., Cheng, X.F., Gong, C.R., Zhou, M., Xu, H.Y., Wei, X.C., Luan, C.N., Pan, D.Y., Li, Z.F., Shi, X.Z. Cryst.Res. Technol. 40 (2005) 882.

[22] Ushasree, P.M., Muralidharan, R., Jayavel, R., Ramasamy,

J. Cryst. Growth 218 (2000) 365.

[23] Ushasree, P.M., Muralidharan, R., Jayavel, R., Ramasamy, J. Cryst. Growth 210 (2000) 741.

[24] Venkataramanan V. Subramanian CK. Bhat HL. J appl Phys 1995; 77:6049-51.

[25] Xing, G., Jiang, M., shao Z., Xu, D. Chin. phys.Lasers 14 (1987) 357. 